\title{
Endoscopic Grading of Adenoid in Otitis Media with Effusion
}

\author{
Acharya K,' Bhusal CL, ${ }^{1}$ Guragain RP' \\ 'Ganesh Man Singh Memorial Academy of ENT and Head Neck studies, Institude of Medicine, Kathmandu,Nepal.
}

\section{ABSTRACT}

Introduction: Adenoid is thought to be one of the causes of otitis media with effusion, though it is controversial. Grading the adenoid by rigid nasal endoscope in patients with Otitis Media with effusion may justify adenoidectomy in Otitis media with effusion in the future.

Methods: A Prospective study was carried out at GMS Memorial Academy of ENT and Head Neck studies from $15^{\text {th }}$ December 2005 - April 2007. Study group comprised of 32 children with otitis media with effusion and control group of 28 children with clinically normal ear and nose. Rigid nasal endoscope was used for grading of adenoid in study and control group. The severity of otitis media with effusion was assessed by preoperative air-bone gap and thickness of the fluid aspirated from middle ear during ventilation tube insertion.

Results: In the study group 13 out of 32 had grade 4 adenoid hypertrophy. This grade 4 adenoid hypertrophy was found to be statistically significant in children with otitis media with effusion $(\mathrm{P}<0.0002)$. In control group 15 out of 28 had grade 1 adenoid hypertrophy which was significant in the same group $(\mathrm{P}<0.002)$. Air - bone gap and thickness of fluid did not correlate with the increasing grade of adenoid hypertrophy.

Conclusions: Grade 4 adenoid hypertrophy was statistically found to be significant with otitis media with effusion but severity of hypertrophy were not reflected by hearing loss and thickness of fluid.

Key Words: adenoid hypertrophy, myringotomy, otitis media with effusion, ventilation tube insertion.

\section{INTRODUCTION}

Adenoid is a pad of lymphoid tissue present at the vault of the nasopharynx. It is considered to be one of the causes of the otitis media with effusion (OME), although it is still controversial.
Nasal endoscopy offers several advantages over the lateral skull radiograph in the evaluation of adenoid hypertrophy. ${ }^{2}$ The grading of adenoid hypertrophy was followed in our study as described by Cassano et al. ${ }^{3}$

\section{Correspondence:}

Dr Kunjan Acharya

Ganesh Man Singh Memorial Academy of ENT and Head Neck studies

Institude of Medicine, Kathmandu,Nepal.

E-mail: acharyakunjan@hotmail.com

Phone: 9851117109 
This particular study was done to put forward new horizon to support the point that adenoid hypertrophy results in otitis media with effusion.

Aims of the study are to grade adenoid by rigid nasal endoscopy in children undergoing myringotomy and ventilation tube insertion and to find out whether there was any correlation between grade of adenoid hypertrophy and severity of otitis media with effusion.

\section{METHODS}

This was a prospective study carried out in Ganesh Man Singh (GMS) Memorial academy of Head and Neck studies, Kathmandu, Nepal from $15^{\text {th }}$ December 2005 to $15^{\text {th }}$ April 2007 (16 months). The sample size for the study was 32 . Patients undergoing ventilation tube insertion for the OME of both genders and age less than or equal to 12 years were included in the study while patients with age more than 12 years, cleft palate, nasopharyngeal mass other than adenoid, dry tap in both ears during myringotomy and sensorineural or mixed hearing loss were excluded from the study.

Children with otitis media with effusion were assessed. The symptoms lasting for more than three months with or without antibiotic therapy in last three months were dated for the surgery. Children who were planned for surgery underwent Tympanometry and Pure Tone Audiometry whenever possible at least one week prior to surgery. Those small children, especially less than five years of age were investigated by the help of play Audiometry.

Children who underwent ENT surgery other than ear and nose were also selected from the operation list of the same day. They were examined for any nasal and ear signs and symptoms and if found normal clinically were selected as control group.

Informed consent was taken. A single dose of intraoperative Ampicillin was given after induction of general anesthesia. Myringotomy was done at anterior-inferior quadrant of pars tensa. The fluid was sucked out and consistency of the fluid regarding its thickness was noted. A Shepard ventilation tube was placed at myringotomy site. The myringotomy without aspiration of fluid was labeled as "dry tap".

After ventilation tube insertion(VTI), choanae was assessed for adenoids by zero degree rigid nasal endoscope. Adenoid was graded based on the grading system described by Cassano et $\mathrm{al}^{3}$ as follows:

Grade1: Choanal opening free, adenoid occupying upper segment of the nasopharynx $(<25 \%$ of choana)
Grade 2: Adenoid tissue occupying upper half of the nasopharynx ( $>=25$ to $<50 \%$ )

Grade 3: Adenoid extending over nasopharynx with obstructing choana and partially the tube $1>=50$ to $<75 \%$ )

Grade 4: Total choanal obstruction ( $>=75$ to $100 \%$ ) Similarly children posted for other surgery in the same day with normal ear and nose was also endoscoped under general anesthesia. These findings were noted and they were taken as the control group.

Data were analysed by the statistician using SPSS 11.5 software. Significance level was assessed by calculating ' $p$ ' value $(<0.005)$ using Pearson's Chi Square test and Fisher exact test.

\section{RESULTS}

There were 32 patients in the study group and 28 patients in control group.

The patients were divided into three age groups: upto 5 years, 6 to 10 yrs and above 10 years to 12 years in both study group and control group. Maximum number of patients was between 6 to 10 years in both groups as shown in following figure.

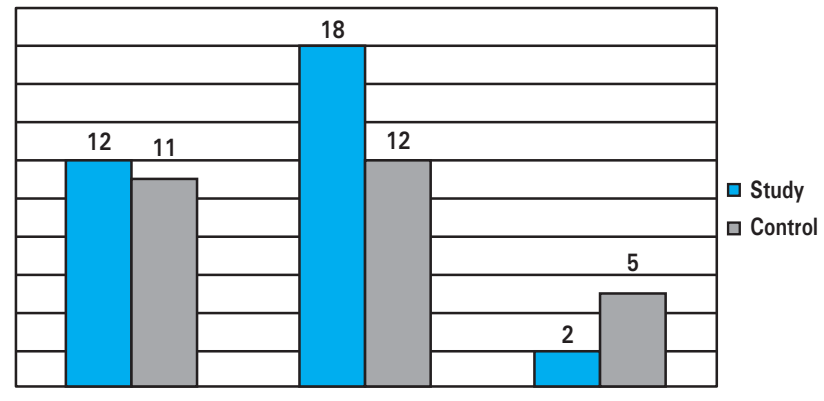

Figure 1. Age distribution

There were 15 males and 17 females in study group and 14 males and 14 females in control group.

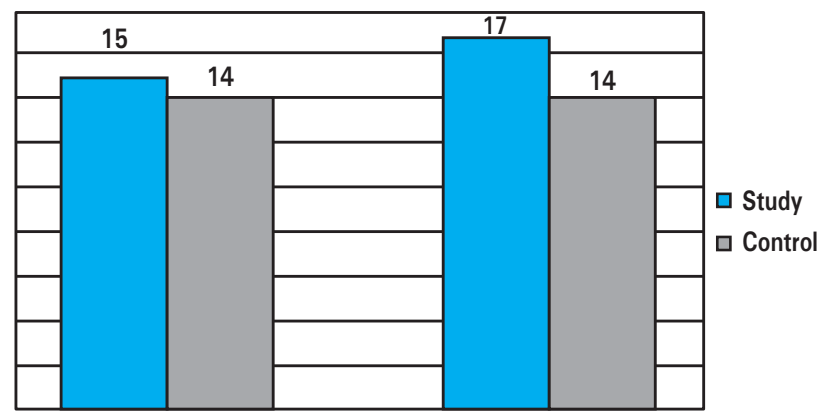

Figure 2. Sex distribution in study and control groups 
Study group had maximum grade 4 adenoid hypertrophy (13/32) followed by grade 3 adenoid hypertrophy (12/32). Control group had maximum of grade 1 hypertrophy i.e, $(15 / 28)$ although grade 4 adenoid was also present in the control group who were not symptomatic on clinical assessment.

Table1. Correlation between different grades of adenoid in study and control group.

\begin{tabular}{llll}
\hline $\begin{array}{l}\text { Grades of } \\
\text { adenoid }\end{array}$ & Study & Groups & P value \\
\hline Grade 1 & 4 & 15 & $0.002^{*}$ \\
Grade 2 & 3 & 6 & 0.281 \\
Grade 3 & 12 & 6 & 0.175 \\
Grade 4 & 13 & 1 & $0.002^{*}$ \\
Total & 32 & 28 & \\
\hline
\end{tabular}

The higher grade of adenoid is more common in study group and lower grade of adenoid in control group.

On futher analyzing the individual grading of adenoid hypertrophy in study and control group, grade 4 adenoid was more commonly found in study group than control group. This means that OME in more commonly encountered in the patients with grade 4 adenoid than rest of the adenoid hypertrophy ( $p=0.002)$. Similarly the control group has more of grade 1 adenoid hypertrophy $(p=0.002)$.

Table 2 shows the distribution of the fluid thickness and also analyzes the grades of adenoid with thickness of fluid. When chi square test was applied thickness of fluid in unilateral as well as bilateral OME was not significantly associated with any grades of adenoid hypertrophy.

\begin{tabular}{|c|c|c|c|c|c|c|c|}
\hline \multicolumn{8}{|c|}{$\begin{array}{l}\text { Table } 2 \text {. Analysis of thickness of fluid in OME with } \\
\text { adenoid hypertrophy }\end{array}$} \\
\hline & & Grade 1 & Grade 2 & Grade 3 & Grade 4 & Total & $P$ value \\
\hline \multirow[t]{3}{*}{ Unilateral } & Thick & 1 & 0 & 4 & 3 & 8 & 0.495 \\
\hline & Thin & 0 & 0 & 0 & 1 & 1 & \\
\hline & Total & 1 & 0 & 4 & 4 & 9 & \\
\hline \multirow[t]{3}{*}{ Bilateral } & Thick & 1 & 2 & 5 & 8 & 16 & 0.302 \\
\hline & Thin & 2 & 1 & 3 & 1 & 7 & \\
\hline & Total & 3 & 3 & 8 & 9 & 23 & \\
\hline
\end{tabular}

For comparison of the hearing with adenoid hypertrophy we calculated air bone gap (A-B gap) in the study group. However, in few cases air bone gap was not possible to calculate because six children had play audiometry for hearing assessment. Hence the sample size for hearing correlation with A-B Gap became 26 (32-6). Table 3 highlights the mean air bone gap in different grades of the adenoid. In none of the grades of the adenoid, the air bone gap was significant. Hence, the severity of hypertrophy of adenoid is not related with air bone gap.

Table 3. Comparison of the air bone gap in individual grading of the adenoids

\begin{tabular}{llllll}
\hline $\begin{array}{l}\text { Grade of Side } \\
\text { adenoid }\end{array}$ & $\begin{array}{l}\text { Min.(A-B } \\
\text { gap) }\end{array}$ & Max(A-B Mean \pm SD & P value \\
\hline grade 1 & Right & 15 & 36 & $28.25 \pm 10.01$ & 0.593 \\
(n=4) & Left & 24 & 41 & $32.00 \pm 8.76$ & \\
Grade 2 & Right & 13 & 33 & $23.0 \pm 14.14$ & 0.415 \\
(n=2) & Left & 30 & 38 & $34.0 \pm 5.66$ & \\
Grade 3 & Right & 15 & 47 & $32.30 \pm 9.20$ & 0.039 \\
(n=10) & Left & 6 & 42 & $22.90 \pm 9.67$ & \\
Grade 4 & Right & 23 & 50 & $38.30 \pm 7.80$ & 0.337 \\
(n=10) & Left & 25 & 51 & $34.80 \pm 8.05$ &
\end{tabular}

\section{DISCUSSION}

Otitis media with effusion (OME) is a common clinical condition affecting young children. Although it is a common disease encountered in the general population, many things about OME regarding its causation and the definite management of the disease is still controversial. Richard Maw in Scott Brown text book of Otorhinolaryngology ${ }^{1}$ stated that $30 \%$ of cases reoccur even after treatment of the offending ear. Similarly he also quoted that ventilation tube produce only short term hearing improvement in speech reception threshold to $20 \mathrm{~dB}$ or less. It is justified from these facts that only ventilation tube insertion does not suffice for the treatment of the otitis media with effusion in all of the cases. So this study was conducted to observe the correlation of the OME with adenoid hypertrophy, as it is thought to be one of the factors responsible for recurrent collection of fluid in the middle ear.

Whenever possible puretone audiogram was performed in the children preoperatively, small and uncooperative children were assessed by play audiometry. Children with sensorineural and mixed hearing loss were excluded from the study because, it may result in biasness while correlating the adenoid hypertrophy with hearing loss. Finally those children who had bilateral dry tap on 
myringotomy were also excluded from the study group as they did not had OME. It accounts for total of five cases. Unilateral dryness of fluid on myringotomy may be due to small amount of the fluid present which could have flown to dependent site like sinus tympani or it may be absorbed due to the use of nitrous oxide during general anesthesia. Hence unilateral ear having glue and dry tap in other were taken for study. Adenoid hypertrophy is believed to play a dual role in etiology of OME. First it may serve as mechanical obstruction to the orifice of the eustachain tube in the nasopharynx, hereby limiting the drainage of the middle ear. ${ }^{4-9}$ Second, they may serve as reservoir for infection, providing a bacterial source for OME. $^{6}$

Our observation showed that increasing grade of adenoid hypertrophy was statistically significant with the otitis media with effusion as ' $\mathrm{P}$ ' value came out to be 0.0002 . On further correlation grade 4 adenoid hypertrophy was only significantly associated with OME. It is strongly supported by the study done by Cassano et al. 3 There are various studies which support adenoidectomy in OME. Kadhim and $\mathrm{Abdul}^{7}$ studied 51,373 children less than 10 years, of which $7.4 \%$ (1096) had pharyngeal surgeries including adenoidectomy and adenotonsillectomy. It showed $39 \%$ less chance of subsequent surgery for OME if they underwent adenoidectomy in first instance by multivariate logistic regression. While Paradise and Bluestone ${ }^{5}$ studied the efficacy of adenoidectomy in 213 children who had received tympanostomy-tube placement because of persistent and/or recurrent otitis media and they had again developed otitis media after tubal extrusion. Statistically significant differences were found mainly in the randomized trial, where during the first and second years, adenoidectomy subjects had $47 \%$ and $37 \%$ respectively less time with otitis media than control subjects and $28 \%$ and $35 \%$ fewer suppurative (acute) episodes than control subjects. They concluded that adenoidectomy was warranted on an individualized basis for children who develop recurrent otitis media after extrusion of tympanostomy tubes.

Here comes the role of grading the adenoids by endoscope so as to avoid thousands of unnecessary adenoidectomy and their attendant risk in OME. In our study as grade 4 adenoid was significantly associated with OME, it may be one of the selection criteria for surgical treatment, although it had to be proven in larger sample size. Our selection criteria is partly supported by the study done by Nguyen and lily ${ }^{8}$ in 63 first instance patients of OME effusion who were randomized into two groups i.e, ventilation tube placement only/and plus adenoidectomy. In both the groups he had performed endoscopy of nasopharynx and commented whether eustachiain tube was obscured by adenoid or not which corresponds to our grading of adenoid of 3 and above. He then followed up the patients for 6 to 12 months. He found that 34 patients in the abutting group/ adenoid obscuring the eustachain tube opening), 16 patients underwent only $\mathrm{VTI}$, of whom 8 (50\%) failed, whereas 18 patients had combined VTI and adenoidectomy, of whom 3 (17\%) failed. There was a statistical difference between these two groups $(P<.05)$. Of the 29 patients in the non abutting group, 24 patients underwent only VTI, of whom $9(37.5 \%)$ failed, whereas 5 patients underwent combined VTI and adenoidectomy, of whom 2 (40\%) failed. There was no statistical difference between these two groups $(P=0.92)$. This study demonstrated that the position of hypertrophied adenoids may alter the final otologic outcome of patients requiring VTI in OME. Patients with adenoids abutting the torus tubaris may benefit most from an adjuvant adenoidectomy in first instance.

Lateral extension of the adenoid resulting in the causation of the otitis media with effusion was also supported by Wright and Alden. ${ }^{9}$ It showed sixty percent of patients undergoing simultaneous VTI were found to have laterally hypertrophied adenoid tissue encroaching upon the ET orifice versus when examined by endoscope during the surgery. Thus, a strong correlation was found to exist between $O M E$, requiring the placement of ventilation tube and lateral adenoids abutting the torus tubarius $\left.X^{2}=39.12 ; P<0.001\right)$. This study in a way supports our finding of grade 4 adenoid hypertrophy and association with OME.

However there is another school of thought, which says that concurrent adenoidectomy does not reduce the incidence of OME in the future. Hammaren and Saxen ${ }^{10}$ evaluated the efficacy of adenoidectomy in reducing the incidence of otitis media among children who are younger than four years. The risk for recurrent otitis media ( $>$ or $=3$ episodes) could not be reduced by adenoidectomy with VTI (odds ratio: $1.66 ; 95 \%$ confidence interval) in a randomized trial conducted at a tertiary center clinic including 217 children.

Maw and Bawdel ${ }^{11}$ showed that after 12 months the mean hearing threshold in 43 children with serous effusion was $19.20 \mathrm{~dB}$ and in 162 with mucoid effusion it was $19.26 \mathrm{~dB}(\mathrm{P}=1.0)$. After two years the mean hearing threshold in the 35 children with serous effusion was $20.52 \mathrm{~dB}$ and in 139 with mucoid effusion it was 18.76 $\mathrm{dB}(\mathrm{P}=0.4)$. These data showed that thickness of fluid had no prognostic significance. In our study also there was no correlection between thickness of fluid and the hypertrophy of adenoid.

Furmann and Goinska ${ }^{12}$ tested 18 children with adenoid hypertrophy and ten healthy children. There was no 
correlation between the middle-ear impedance and the value of hearing threshold level for $500 \mathrm{~Hz}$ and average value for 500,1000 and $2000 \mathrm{~Hz}$ after adenoidectomy in eight children. We also calculated mean of A-B Gap in all study groups in same frequency $(500,1000,2000$ $\mathrm{Hz}$ ) and correlated with adenoid hypertrophy, which showed no statistical significance.

\section{CONCLUSIONS}

Higher grades of adenoid hypertrophy assessed by rigid nasal endoscope was associated significantly in children with otitis media with effusion than in control groups with clinically normal ear and nose $(P<0.0002)$. On further analysis, grade 4 hypertrophy was the only grade of adenoid found to be statistically significant in children with otitis media with effusion $(P<0.002)$. But the severity of adenoid hypertrophy did not correlate with the thickness of fluid and air bone gap. Thus children with grade 4 adenoid hypertrophy may be considered as one of the selection criteria for the adenoidectomy in otitis media with effusion. However it has to be proven by further randomized clinical trials in future with larger sample size and long term follow up.

\section{REFERENCES}

1. Maw RW. Otitis media with effusion. In: Scott- Brown's Otolaryngology. 6th edition, Great Britian: ButterworthHeinemann;1997. p. 6/7/3 -6/7/10.

2. Parikh SR, Coronel M, Lee JJ. Validation of a new grading system for endoscopic examination of adenoid hypertrophy. Otolaryngol - Head and Neck Surg. 2006;135(5):684-7.

3. Cassano P, Gelardi M, Caszsano M. Adenoid tissue rhinopharyngeal obstruction grading based on fiberendoscopic findings: a novel approach to therapeutic management. Int J Pediatr Otolaryngol. 2003;67(12):1303-9.

4. Maw AR. Chronic otitis media with effusion (glue ear) and adenotonsillectomy. BMJ. 1983;287:1586-8.

5. Paradise JL, Bluestone CD, Rogers KD. Efficacy of adenoidectomy for recurrent otitis media: results from parallel random and non-randomized trials. JAMA. 1990;263:2066-73.

6. Okur E, Yildirim I, AkifKilic M, Guzelsoy S. Prevalence of otitis media with effusion among primary school children in Kahramanmaras, in Turkey. Int J Pediatr Otorhinolaryngol. 2004;68(5):557-62.
7. Kadhim, Latif A, Spilsbury. Adenoidectomy for Middle Ear Effusion: A Study of 50,000 Children Over 24 Years. Laryngoscope. 2007;117(3):427-33.

8. Nguyen, Lily HP, Manoukian, John J. Adenoidectomy: Selection Criteria for Surgical Cases of Otitis Media. Laryngoscope. 2007;117(3):427-9.

9. Erin DW, Alden JP, John JM. Laterally hypertrophic adenoids as a contributing factor in otitis media. Int J of Pediatr Otorhinolaryngol. 1998;45(3):207-14.

10. Hammarén MS, Saxen H, Tarkkanen J. Adenoidectomy does not significantly reduce the incidence of otitis media in conjunction with the insertion of tympanostomy tubes in children who are younger than 4 years: a randomized trial. Pediatr. 2005 ;116(1):185-9.

11. Maw RA, Bawdel R, Keefe LO et al. Does the type of middle ear aspirate have any prognostic significance in otitis media with effusion in children? Clin Otolaryngol. 1993;18:396-9.

12. Furmann A, Goinska A, Hojan E. Effect of middle ear impedance on hearing threshold level in children with adenoid hypertrophy. Otolaryngol Pol. 2002;56(1):77-81. 